Revue de l'Institut des langues et cultures

d'Europe, Amérique, Afrique, Asie et Australie

$11 \mid 2009$

Langues \& cultures de spécialité à l'épreuve des médias

\title{
L'impact des attentats du 11 septembre 2001 sur le discours relatif aux immigrants dans la presse canadienne et australienne
}

Sandrine Tolazzi et Carole Maserati

\section{CpenEdition}

\section{Journals}

Édition électronique

URL : http://journals.openedition.org/ilcea/76

DOI : 10.4000/ilcea.76

ISSN : 2101-0609

Éditeur

UGA Éditions/Université Grenoble Alpes

Édition imprimée

ISBN : 978-2-84310-179-3

ISSN : 1639-6073

Référence électronique

Sandrine Tolazzi et Carole Maserati, «L'impact des attentats du 11 septembre 2001 sur le discours relatif aux immigrants dans la presse canadienne et australienne », ILCEA [En ligne], 11 | 2009, mis en ligne le 30 avril 2009, consulté le 08 mars 2021. URL : http://journals.openedition.org/ilcea/76 ; DOI : https://doi.org/10.4000/ilcea.76

Ce document a été généré automatiquement le 8 mars 2021

(c) ILCEA 


\title{
L'impact des attentats du
} 11 septembre 2001 sur le discours relatif aux immigrants dans la presse canadienne et australienne

\author{
Sandrine Tolazzi et Carole Maserati
}

\section{NOTE DE L'AUTEUR}

Par souci de lisibilité, les mots en anglais ont été mis en italique dans le texte et les citations traduites en français par les auteures.

1 Si les attentats du 11 septembre 2001 ont entraîné un traumatisme psychologique et des répercussions politiques et économiques immédiates, l'intensité et la durée de l'onde de choc provoquée par ces attentats sont elles aussi révélatrices de l'impact de cet événement à l'échelle mondiale. De nombreux journalistes et analystes politiques occidentaux, relayés par les médias, continuent aujourd'hui à envisager les faits saillants de l'actualité à travers le prisme de ce «choc des civilisations » qui a marqué l'entrée d'un monde peut-être pas encore tout à fait global dans le troisième millénaire. On peut donc constater depuis ces attentats un changement du discours journalistique qui s'avère plus ou moins visible selon les pays où est produit ce discours et selon les domaines concernés.

2 Cette étude a cherché à identifier un tel changement au niveau de la presse canadienne et australienne en ce qui concerne le discours relatif aux immigrants. L'aspect comparatif semble pertinent dans la mesure où le Canada et l'Australie, culturellement très proches des États-Unis, ont tous deux vécu le 11 septembre de manière particulièrement intense. Au lendemain des attaques, tandis que le Premier ministre canadien Jean Chrétien offrait son soutien en Afghanistan à son plus proche voisin et partenaire économique, son homologue australien John Howard, alors en visite 
officielle aux États-Unis, s'empressait de renforcer les liens existants en proposant un appui militaire à George W. Bush. L'Australie, qui s'est engagée par la suite dans la guerre en Iraq, aura d'ailleurs à en payer la facture avec les attentats de Bali en octobre $2002^{1}$, mais se verra également récompensée par la signature d'un traité de libre-échange avec les États-Unis qui entrera en vigueur en janvier 2005. D'autre part, ces deux pays présentent également des similitudes en ce qui concerne leur politique d'immigration : d'abord méfiants vis-à-vis des immigrants non européens, ils se sont petit à petit tournés vers des sources d'immigration de plus en plus diversifiées tout en établissant une sélection fondée non plus sur des critères ethniques mais socioéconomiques. À l'heure où les mouvements de populations se font de plus en plus importants et où les États doivent trouver un équilibre entre leurs intérêts et la dimension humanitaire dans leurs programmes d'immigration, le Canada et l'Australie mettent tous deux l'accent sur une immigration choisie composée en grande majorité de travailleurs qualifiés. S'ils réservent un certain nombre de places pour les réfugiés, ils font de la lutte contre l'immigration dite "illégale » une priorité.

En gardant à l'esprit ce rapprochement qui peut être fait entre les deux pays, nous nous sommes penchées sur les articles de deux journaux caractéristiques du «discours dominant", The Globe and Mail (Canada) et The Australian (Australie), afin de tester l'hypothèse d'un changement du discours relatif aux immigrants suite aux événements du 11 septembre 2001. Pour ce faire, nous avons isolé tous les articles mentionnant le terme immigrants parus entre le 11 mars 2001 et le 11 mars 2002, soit 6 mois avant et 6 mois après le 11 septembre $2001^{2}$. L'étude de ce corpus de 490 articles canadiens et 389 articles australiens nous a permis de déterminer dans quelle mesure les événements du 11 septembre 2001 venaient se superposer au discours dominant sur les immigrants. Nous verrons donc dans un premier temps quelle est la nature de ce discours dominant produit par chacun des deux quotidiens concernés tout au long de la période étudiée. Ensuite, nous mettrons en valeur les transformations qui peuvent être interprétées comme une conséquence du 11 septembre 2001. Enfin, nous tenterons de modérer ce propos en montrant comment le contexte dans lequel s'inscrit le discours sur les immigrants reste un élément prépondérant dans les deux pays.

\section{Le discours dominant}

\section{Le Canada : renforcement des frontières, réfugiés et immigrants «illégaux »}

The Globe and Mail, sur lequel se focalise cette étude du discours sur les immigrants au Canada, est le quotidien national le plus populaire du pays. Il a été créé en 1936, suite à la fusion entre The Globe, journal hebdomadaire du parti libéral depuis 1844, et The Mail and Empire, fondé en 1872 par John A. Macdonald et organe de presse du parti conservateur. Il est maintenant considéré comme un journal de référence, sérieux et non engagé. Les 490 articles contenant le terme immigrants sur la période du 11 mars 2001 au 11 mars 2002 ont été écrits par un large éventail de journalistes, et très peu de noms se retrouvent d'un article à l'autre. On compte toutefois quelques articles écrits par des personnalités extérieures, comme le sociologue Martin Weinfield, l'ancien ambassadeur du Canada en Yougoslavie James Bissett (qui fut également à la tête du 
Service d'Immigration Canadienne de 1985 à 1990), ou encore Sharryn Aiken, membre du Centre d'études sur les réfugiés de l'Université de York à Toronto.

Le thème du contrôle des frontières est un thème récurrent tout au long de la période concernée. Ceci provient du fait que le gouvernement, en 2001, essaie de faire entériner le projet de loi C-11, autrement dit la Loi sur l'immigration et la protection des réfugiés, qui doit remplacer la Loi sur l'immigration de 1976 en redéfinissant les critères de sélection des immigrants. Jugé trop strict par certains et pas assez par d'autres, ce nouveau projet de loi ainsi que les débats qui l'accompagnent sont l'objet d'une attention constante de la part des journalistes : «La Chambre des Communes a voté le projet de loi C-11 au mois de juin [2001] pour renforcer nos lois sur l'immigration, ce qui a donné lieu à des contestations de la part des groupes de défense des libertés civiles qui trouvaient que le gouvernement s'octroyait trop de pouvoirs arbitraires dans le but de refuser et expulser les visiteurs et les immigrants $\|^{3}$. Les articles relatent très fréquemment les critiques faites par l'opposition à la ministre de l'Immigration, Elinor Caplan, mais aussi, dans un souci d'équilibre, les arguments de celle-ci en faveur du texte: « $\mathrm{M}^{\mathrm{me}}$ Caplan a répété hier que selon elle, de toute évidence, les Canadiens voulaient un gouvernement plus sévère envers les immigrants illégaux ou ayant enfreint la loi, ainsi qu'une simplification des procédures dans le but de procéder plus rapidement à leur expulsion. $»^{4}$

6 Les points d'achoppement concernent donc principalement les immigrants dits « illégaux », qui sont fréquemment assimilés aux réfugiés et parfois liés dans les articles à la criminalité : une recherche dans l'ensemble des articles avec illegal immigrants et crime puis avec illegal immigrants et criminal(s) donne respectivement quatre et cinq réponses avant le 11 septembre et six et huit après le 11 septembre. En associant les mots refugees et crime puis refugees et criminal(s), on trouve un nombre conséquent d'articles : dix-huit et quinze avant les attentats, seize et vingt et un après. Il y a donc une tendance récurrente dans le discours du Globe and Mail à faire un lien entre la criminalité et les immigrants non choisis par le Canada, et à faire passer ces derniers pour des individus potentiellement dangereux, créant ainsi un amalgame dans l'esprit du lecteur.

7 Pourtant, en parallèle, on trouve à plusieurs reprises des articles critiquant le refus de l'Australie de laisser entrer sur son territoire des réfugiés afghans, immigrants dits «illégaux » car ils ne sont pas passés par la procédure d'immigration classique mais projetaient de faire une demande d'asile une fois les côtes australiennes atteintes. Ces articles mentionnent notamment le placement automatique en centre de détention de ces immigrants, et citent l'exemple d'un enfant iranien enfermé depuis dix-sept mois et souffrant de troubles post-traumatiques ${ }^{5}$. L'insensibilité du ministre australien de l'Immigration, Philip Ruddock, est dénoncée ici, puisqu'il affirme que la détention est la seule possibilité pour pouvoir ensuite expulser les immigrants «illégaux»: «Si on laissait les gens libres au sein de la communauté... est-ce qu'ils se présenteraient d'euxmêmes le jour de leur expulsion? ». Plutôt que de soutenir cette position, le journaliste donne la parole à des opposants au placement en détention des immigrants « illégaux ", qui craignent que le traitement de la demande d'asile prenne plusieurs années, pendant lesquelles les 2500 immigrants «illégaux» (dont 500 enfants) resteraient enfermés dans ces centres de détention. Néanmoins, le journaliste Marcus Gee écrit qu'il est facile de condamner l'attitude du gouvernement australien mais qu'il ne faut pas 
oublier que le gouvernement canadien a fait, dans un passé encore proche, exactement la même chose :

Les Canadiens, tolérants, compatissants et bienveillants, hocheront la tête en signe de réprobation devant le traitement brutal que l'Australie réserve aux boat people afghans. [...] Évidemment, notre pays ne renverrait jamais un bateau rempli de réfugiés désespérés. Sauf que nous l'avons fait. En 1914, le Canada a renvoyé un bateau entier de Sikhs indiens qui avait attendu deux mois dans le port de Vancouver à bord du Komagata Maru. Dans les années 1930, il a renvoyé des milliers de réfugiés juifs fuyant l'Allemagne nazie. Et même pas plus tard qu'en 1999, de nombreux Canadiens ont demandé au gouvernement de rejeter les bateaux de réfugiés chinois qui commençaient à arriver sur la côte ouest. ${ }^{6}$

8 Il conclut: «Dans un monde où le fossé entre riches et pauvres est aussi énorme, il y aura toujours des malheureux frappant à notre porte. Tout ce que nous pouvons faire est de les traiter avec équité et décence. $»^{7}$

Ceci amène les journalistes à faire un constat sur la contradiction entre le besoin d'immigrants qu'ont les pays développés au niveau démographique et leur refus d'accepter une bonne partie d'entre eux : «Ceci est une atteinte au bon sens. À cause d'un taux de fécondité en baisse, les pays occidentaux ont une population qui comporte de plus en plus de personnes à la retraite et de moins en moins d'actifs. [...] Une immigration légale aiderait à combler cet écart démographique. $»^{8}$ Toutefois, le journaliste finit par nuancer: "Même si les pays occidentaux voulaient ouvrir leurs portes à une immigration légale, ils ne pourraient pas recevoir tous les individus qui souhaiteraient vivre chez eux $»$.

\section{L'Australie : immigrants «illégaux ", passeurs et centres de détention}

10 Avant de faire ressortir les principales caractéristiques du discours sur les immigrants dans The Australian, il convient de souligner que ce journal est le seul quotidien national australien', même s'il est très fortement concurrencé par les quotidiens des grandes métropoles comme le Sydney Morning Herald ou The Age (Melbourne). Lancé par le magnat des médias Rupert Murdoch en 1964, il est décrit par Chris Mitchell - son rédacteur en chef - comme un journal de centre-droite, ce qui ne l'empêche pas de publier régulièrement des articles rédigés par des auteurs résolument de gauche, comme Phillip Adams ${ }^{10}$. Parmi les 389 articles contenant le mot « immigrants » parus dans ce journal entre le 11 mars 2001 et le 11 mars 2002, une attention toute particulière a été accordée aux articles signés par des auteurs de renom, tels que l'auteur précité ou encore Robert Garran ${ }^{11}$, par les rédacteurs des rubriques politiques, affaires étrangères ou affaires nationales (Denis Shanahan, Greg Sheridan, Mike Steketee), ou encore par des journalistes traitant régulièrement du sujet (Megan Saunders, Claire Harvey). Une recherche par mots-clés sur l'ensemble des articles ainsi qu'une analyse plus fine de certains d'entre eux permet de faire ressortir les aspects les plus importants du discours sur les immigrants véhiculés par ce journal entre le 11 mars 2001 et le 11 mars 2002.

11 Tout d'abord, on note une distinction très nette entre les immigrants rigoureusement sélectionnés en fonction de leur profil socio-économique - et donc susceptibles de contribuer à la société australienne en s'intégrant rapidement dans le monde du travail - et ceux qui souhaitent obtenir le statut de réfugiés, présentés comme beaucoup moins 
désirables. Le journal note par exemple les changements en matière de politique d'immigration en soulignant que l'augmentation de la première catégorie d'immigrants fait l'unanimité («Le gouvernement fédéral a annoncé que 6000 immigrants supplémentaires (immigrants qualifiés et réunion familiale) pourraient entrer en Australie, décision accueillie favorablement par l'opposition et le monde des affaires. [...] Cette augmentation était nécessaire pour permettre à la population australienne d'atteindre les 24 millions visés par le gouvernement. $»^{12}$ ). En revanche, le même article ajoute qu'il n'y aura aucun changement au niveau des programmes d'accueil des réfugiés, déplorant en outre le fait qu'« on s'attend à davantage de demandes concernant les immigrants illégaux, à moins que les tentatives du gouvernement de mettre fin au trafic de clandestins ne portent leurs fruits. ${ }^{13}$. C'est bel et bien sur cette dernière catégorie d'immigrants que se focalise le discours de The Australian, puisque sur les 172 articles concernant la période antérieure au 11 septembre et les 217 suivant les attentats, on compte 105 articles (61\%) dans le premier cas et $101(46 \%)$ dans le second faisant référence aux "immigrants illégaux " (illegal immigrants). Qui sont ces immigrants illégaux régulièrement mentionnés par le journal? Dans plus de $60 \%$ des cas, l'article contenant le terme illegal immigrants emploie conjointement les mots refugee(s), boat people (ou boatpeople) ainsi que asylum seeker(s) (64 articles avant le 11 septembre, 66 après le 11 septembre). Cette association semble renforcer l'hypothèse selon laquelle exilés, réfugiés ou demandeurs d'asile ne sont guère les bienvenus sur le sol australien. Si l'on y regarde de plus près, la plupart de ces articles font mention des immigrants arrivés par bateau sur le territoire grâce à l'aide de passeurs.

Le trafic de clandestins (people-smuggling) est donc également un thème central du discours de The Australian qui se retrouve dans $21 \%$ des articles publiés avant le 11 septembre et $18 \%$ des articles publiés après le 11 septembre ${ }^{14}$. Dans chacune des deux périodes concernées, ce trafic est présenté comme une véritable menace pour l'Australie. Les passeurs sont décrits (à juste titre) comme des criminels participant à la troisième plus grande activité du crime organisé après le trafic d'armes et de drogue. Un article publié en juin 2001 s'indigne des « dizaines de millions de dollars » amassés par les plus importants d'entre eux et donne l'exemple d'un passeur installé en Indonésie qui aurait gagné 40 millions de dollars en quelques années ${ }^{15}$. Deux mois plus tard, l'image de l'Australie auprès de la communauté internationale est mise à rude épreuve alors que le pays refuse le droit au navire Tampa de débarquer des naufragés sur son territoire, contrairement aux lois internationales en vigueur. Cela n'empêche pourtant pas le journal de considérer que la réaction du gouvernement "peut être justifiée » et, plutôt que de se pencher sur le sort des 430 personnes sauvées des eaux, de rappeler que :

Le trafic illégal d'êtres humains à travers les frontières représente désormais une industrie mondiale estimée à 20 milliards de dollars en 1999. En Europe, le nombre de demandeurs d'asile excède depuis longtemps le nombre de travailleurs étrangers entrés de manière légale. En Australie, ce n'est pas simplement l'intégrité de notre programme concernant les réfugiés qui est en danger; selon le gouvernement Howard, c'est la souveraineté nationale de l'Australie. ${ }^{16}$

C'est pourquoi The Australian fait souvent grand cas des passeurs arrêtés par les autorités australiennes, comme cet Irakien accusé en octobre 2001 d'avoir facilité l'entrée illégale de 1698 personnes au moyen de 17 bateaux depuis 1999, permettant ainsi à son réseau de bénéficier de revenus substantiels puisqu'en règle générale, chaque passager devait débourser 5000 dollars américains ${ }^{17}$. Les chiffres, qu'ils fassent 
référence aux sommes apparemment astronomiques que gagnent les passeurs ou au nombre important d'immigrants illégaux arrivés majoritairement par ce biais (plus de 11500 depuis 1989 et 7600 depuis 1999, indique l'article de juin ${ }^{18}$, tandis qu'un article d'octobre 2001 fait référence aux 13500 immigrants illégaux arrivés au cours des 10 années ${ }^{19}$ précédentes) donnent du poids à un discours qui alimente la peur de ce type d'immigrants et la haine des passeurs, et qui fait apparaître la lutte contre une telle immigration comme un élément indispensable à la sauvegarde de la souveraineté australienne.

Le journal semble donc vouloir soutenir la politique du gouvernement en ce qui concerne ces immigrants «illégaux ». En référence à la crise du Tampa, il donne son appui à une décision dictée, selon lui, par la raison et critique à la fois les Australiens qui s'y opposent et la communauté internationale qui la dénonce. Il dépeint par ailleurs ces immigrants comme des calculateurs qui manipulent les autorités en "perdant» volontairement leurs papiers d'identité :

Pourquoi les demandeurs d'asile se débarrassent-ils de leurs papiers avant d'arriver dans le pays où ils souhaitent demander l'asile? De toute évidence, ils veulent dissimuler leur pays d'origine. Certains pays présentent en effet davantage de raisons de s'exiler que d'autres. La dissimulation d'identité est un véritable cassetête pour les services d'immigration. Car tout ce qui est caché n'est pas innocent. ${ }^{20}$

Partant de là, The Australian approuve en grande partie le refus du gouvernement Howard d'accepter les passagers du Tampa sur le sol australien et, en ce qui concerne les immigrants clandestins qui parviennent à atteindre les côtes australiennes, il soutient la mesure qui consiste à les enfermer dans des camps de détention en attendant le traitement de leur demande.

Cette question des centres de détention constitue, en dernier lieu, un autre thème qui ressort dans $31 \%$ des articles publiés avant le 11 septembre 2001, et $24 \%$ des articles publiés après. Il est vrai que compte tenu du nombre conséquent de demandeurs d'asile qui arrivent sur les côtes australiennes, la pression sur ces centres est de plus en plus forte, ce qui conduit à de multiples émeutes et grèves de la faim parmi les détenus et à des manifestations de soutien organisées par de nombreux intellectuels et activistes. Là encore, le journal fait le choix de ne pas s'appesantir sur les conditions de vie dans les centres, si ce n'est pour se faire le relais des éventuelles solutions soutenues par le Conseil australien des libertés civiles (port d'un bracelet électronique pour éviter la détention) ou le Parti travailliste d'opposition (création d'un centre sous haute sécurité pour les immigrants dont le dossier a été rejeté) ${ }^{21}$. En revanche, il semble à nouveau mettre en relief le nombre d'immigrants illégaux et le danger qu'ils représentent :

Les dernières informations nous parvenant d'Indonésie suggèrent que des centaines d'autres boat people se dirigent vers l'Australie; les autorités craignent qu'il ne suffise que de quelques bateaux pour que les centres de détention n'atteignent leurs limites en terme de capacité. [...] Le ministère de l'Immigration a fait référence aux problèmes de santé de certains détenus - un cas de typhoïde, un cas de paludisme, un cas de méningite à méningocoque et quatre cas de rougeole - pour justifier le régime de détention obligatoire. ${ }^{22}$

$\mathrm{Si}$, comme nous allons le voir, le journal sera plus prudent en ce qui concerne ses positions après le 11 septembre 2001, faisant preuve de davantage de compassion, il considère néanmoins que les centres de détention sont, au pire, insuffisants pour dissuader les immigrants illégaux et, au mieux, un mal nécessaire. Certains journalistes n'hésitent donc pas à critiquer des collègues un peu trop prompts à s'opposer à la 
politique de détention du gouvernement et à dénoncer le racisme latent de la population : ${ }^{23}$

J'aimerais faire une distinction entre la dimension humanitaire qu'il convient d'appliquer à la politique d'immigration à long terme et le débat concernant les demandeurs d'asile. Certaines personnes - notamment Phillip Adams et d'autres partisans de sa campagne de désobéissance à la Ghandi - ont une vision particulière de ce débat, dictée par les événements actuels et le prisme étroit de leurs opinions politiques. Ce mouvement, peu important mais bruyant, semble disposé à laisser entrer quiconque en Australie, et considère immédiatement que toute personne soutenant le principe de détention des demandeurs d'asile ainsi qu'une politique qui cherche à distinguer les réfugiés réels des personnes voulant prendre un raccourci sur le chemin d'une vie meilleure est sectaire, intolérante, raciste, et manque de compassion. ${ }^{24}$

Dans tous les cas, The Australian se fait le relais d'une opinion publique majoritairement opposée à toute immigration "illégale », à moins qu'il ne banalise tout simplement la haine de l'autre en rapportant allégrement, par exemple, les propos de «M. Toutlemonde » au sujet des boat people: « explosez leur bateau»; «coulez-le»; «ils devraient avoir un 747 à l'aéroport international de Perth pour les reconduire directement d'où ils viennent "; «ils sont traités comme des seigneurs "; «ils nous coûtent des millions $» .25$

Lorsqu'on étudie les articles mentionnant le terme immigrants sur une période d'un an 6 mois avant le 11 septembre 2001 et 6 mois après - on remarque donc plusieurs similitudes dans le discours de The Globe and Mail et The Australian. Tout d'abord, les deux journaux se focalisent sur les réfugiés ou les immigrants qui parviennent à entrer clandestinement sur les territoires canadien et australien. C'est ce type d'immigration qui pose problème aux gouvernements des pays concernés. The Australian met l'accent sur la lutte sans relâche menée contre ceux qui arrivent par milliers sur les côtes australiennes et soutient la politique qui consiste à les enfermer dans des centres de détention en attendant le traitement (et bien souvent le rejet) de leur demande, tandis que The Globe and Mail critique ces mesures et se montre un peu plus modéré en intégrant différents points de vue. Mais dans les deux cas, c'est bien l'immigration «illégale» qui semble dominer dans le discours sur les immigrants. Nous allons cependant voir qu'au-delà de cette constante, ce discours tend à se transformer après le 11 septembre 2001.

\section{Des changements importants}

\section{Immigrants « illégaux » et réfugiés : une menace pour le Canada}

Comme l'on pouvait s'y attendre, les attentats du 11 septembre ont modifié le regard que portaient les journalistes sur les immigrants. En effet, alors que le Canada accueille chaque année plus de 200000 d'entre eux, ils sont davantage considérés comme des menaces potentielles pour le pays après septembre 2001. En témoigne une recherche dans les 490 articles du corpus qui trouve 108 résultats en associant le mot immigrants à terrorist(s), ou terrorism dans la période après le 11 septembre, alors qu'elle n'en obtenait que neuf avant les attentats. Le premier changement concerne les commentaires sur la nouvelle politique d'immigration définie par la Loi sur l'immigration et la protection des réfugiés. Les journalistes passent de la critique (ou du moins de l'exposition des arguments de l'opposition) au soutien appuyé au gouvernement, voire 
même à l'accusation selon laquelle il n'est pas assez strict. Ainsi, ils étaient dans un premier temps assez prompts à dénoncer une loi qui attribuait selon eux un pouvoir trop important aux responsables de l'immigration concernant l'expulsion d'individus qui se trouvent au Canada depuis des années ${ }^{26}$, ou du moins à exposer les arguments de la ministre de l'Immigration mais aussi du parti d'opposition ${ }^{27}$. En revanche, après les attentats, la critique contre le gouvernement se fait toujours acerbe, mais cette fois dans le sens inverse: le gouvernement est accusé de ne pas être assez dur en ce qui concerne le contrôle des frontières, et de ne pas octroyer de moyens (financiers ou humains) suffisants pour que le contrôle soit vraiment efficace. Un spécialiste du contre-terrorisme interrogé par un journaliste du Globe and Mail énumère tous les points faibles du système de contrôle des frontières et ajoute que « la sécurité n'est pas une priorité importante pour les Canadiens ${ }^{28}$. Plus loin, le journaliste poursuit :

Lorsque je lui demande si nous sommes en sécurité à présent, M. Riberdy me fait reformuler ma question: est-ce que nous sommes plus en sécurité maintenant? «Oui, nous sommes plus en sécurité maintenant» répond-il. Mais, comme beaucoup de Canadiens depuis le 11 septembre, je ne veux pas savoir si nous sommes plus en sécurité. Je veux juste savoir si nous sommes en sécurité.

Or, nous avons vu dans la première partie que les journalistes associaient l'idée d'une menace aux immigrants dits «illégaux» ou aux réfugiés. C'est toujours le cas dans la période qui suit le 11 septembre, mais à cela il faut ajouter le lien qui est établi entre les immigrants illégaux et le terrorisme : alors qu'aucun article ne crée un tel lien avant les attentats, une recherche entre septembre 2001 et mars 2002 nous donne vingt-sept réponses. Cela traduit un durcissement de la ligne éditoriale envers cette catégorie d'immigrants. En d'autres termes, la cible de la critique devient le système d'accueil des réfugiés et par extension l'incapacité du gouvernement à contrôler ses frontières de manière efficace.

Avant le 11 septembre, le principal message que font passer les journalistes est que le système d'accueil des réfugiés n'est pas adapté à la réalité des besoins, dans le sens où il laisse entrer des individus dangereux tout en en expulsant d'autres de manière totalement injuste. En effet, quelques articles se focalisent sur l'exemple d'une famille d'origine polonaise (les parents et leurs quatre enfants dont deux sont nés au Canada) qui est renvoyée dans son pays d'origine car elle a par inadvertance payé 1150 \$ au lieu de 1200 \$ pour sa demande d'asile. Les critiques sont ici assez dures envers la ministre de l'Immigration, "the arrogant Ms. Caplan $»^{29}:$ "there is no justification for what followed». Par opposition, le journaliste John Saunders mentionne que le Canada a laissé entrer un ressortissant allemand appartenant à un groupe terroriste dans les années $1980^{30}$. Un des thèmes développés dans cette période précédant le 11 septembre est la demande d'amnistie pour tous les immigrants illégaux : les journalistes se font le relais des différents points de vue mais donnent davantage la parole à l'opposition qui souhaite que tous ces individus soient régularisés. Le journal cite les deux exemples que sont à la fois les États-Unis ${ }^{31}$ et le Portugal qui ont donné une amnistie aux immigrants illégaux dans leur pays. Il relate les difficultés d'être immigrant illégal au Canada par des exemples précis, comme celui de Mary, une femme de ménage originaire des îles Grenadines, arrivée au Canada en 1990 pour fuir son mari et offrir davantage d'opportunités à son fils, et à qui une demande d'asile a été refusée ${ }^{32}$.

Après le 11 septembre, le discours est tout autre: on ne s'attarde plus sur les victimes d'un système auparavant considéré comme trop dur et parfois injuste, mais les critiques portent alors sur ce système considéré par beaucoup comme trop souple et 
laissant par conséquent entrer des individus susceptibles de constituer une menace pour le pays. James Bissett, ancien ambassadeur du Canada en Yougoslavie et ancien directeur du Service canadien de l'immigration, dénonce l'«irresponsabilité »du gouvernement ${ }^{33}$ :

Les porte-parole du gouvernement saluent la nouvelle loi sur l'immigration actuellement discutée au Sénat pour sa sévérité à l'encontre des terroristes et des criminels. Pourtant, cette nouvelle loi, C-11, ne couvre pas le domaine dans lequel le Canada est le plus vulnérable: notre système d'accueil des réfugiés, inadapté et bien trop généreux. Le projet de loi C-11 facilitera l'entrée au Canada des demandeurs d'asile et rendra beaucoup plus difficile leur expulsion une fois qu'ils seront entrés. Dans des circonstances normales, cet échec serait déplorable. Après le 11 septembre, le refus de mettre en place une réforme immédiate de notre système relatif aux demandeurs d'asile relève de l'irresponsabilité criminelle.

Ce sont donc bien les réfugiés et demandeurs d'asile qui sont pointés du doigt dans l'après 11 septembre. Alors qu'un article écrit avant les attentats par le sociologue Martin Weinfield ${ }^{34} \mathrm{~s}^{\prime}$ attachait à dénoncer tous les stéréotypes en général attribués aux réfugiés, l'ancien ambassadeur James Bissett énumère et légitime désormais dans un article d'octobre $2001^{35}$ toutes les critiques que l'on note habituellement à leur encontre: ils peuvent faire une demande d'asile même s'ils n'ont pas de papiers, ils prennent la place de " vrais » réfugiés, ils présentent un risque potentiel et menacent l'intégrité du pays. Un groupe d'individus se disant spécialistes du système d'accueil des réfugiés écrit ${ }^{36}$ :

Quel danger représentent quelques immigrants illégaux dans un pays aussi grand et aussi riche que le Canada? Après tout, nous sommes une nation sincèrement humanitaire, n'est-ce pas? Eh bien, non. [...] Les tricheurs et les fraudeurs, les trafiquants, les profiteurs et les opportunistes sont maintenant aux commandes de notre système d'immigration. Résultat: dommage pour les enfants déplacés d'Afghanistan. Nous n'avons ni le temps ni l'argent pour les aider. Et ceci n'est pas l'opinion d'une bande de cinglés de droite anti-immigrants. C'est la conclusion réfléchie et déchirante de nombreuses personnes très expérimentées et aux vues libérales qui connaissent intimement le système.

Par conséquent, de nombreux articles évoquent la nécessité d'harmoniser les lois relatives au contrôle des frontières avec celles des États-Unis afin de créer un périmètre de sécurité empêchant de laisser entrer les immigrants susceptibles de représenter une menace pour le pays. Cette décision est principalement motivée par des raisons économiques, les États-Unis étant un des partenaires majeurs du Canada, et par le fait que la frontière entre les deux pays ne semble pas défendue : «Le Canada et les États-Unis partagent la plus longue frontière non-défendue du monde. Elle mesure 8892 kilomètres. Les deux pays ont également les relations économiques bilatérales les plus conséquentes au monde, relations qui excèdent le million de dollars américains par jour. $»^{37}$ On trouve beaucoup d'articles dans lesquels les États-Unis enjoignent au Canada d'agir en faveur d'un renforcement des frontières, sous peine d'encourir une baisse significative de leurs importations et exportations. Les journalistes citent fréquemment des responsables américains poussant le gouvernement canadien dans cette voie (comme John Ashcroft, ministre américain de la Justice ${ }^{38}$, ou bien Paul Cellucci, ambassadeur des États-Unis au (anada ${ }^{39}$ ). Par la même occasion, ces journalistes critiquent le refus du Canada de s'exécuter. 


\section{Le paradoxe de The Australian}

26 La première hypothèse à vérifier en ce qui concerne le discours de The Australian après le 11 septembre est également celle d'une peur croissante des immigrants, les attaques ayant montré que la menace terroriste pouvait venir de l'intérieur même d'un pays par le biais d'individus opposés à ses valeurs. Cette hypothèse se confirme à première vue, puisque parmi les articles publiés avant le 11 septembre, seuls deux associent le mot immigrants aux mots terrorism ou terrorist(s); en revanche, 53 articles publiés après le 11 septembre font la même association. Or, quel que soit le contexte ou le sujet de ces articles, la présence de ces termes a pour conséquence leur mise en relation dans l'esprit du lecteur et alimente donc la crainte de l'Autre - cet « Autre » étant d'ailleurs bien souvent l'immigrant du Moyen-Orient. Déjà stigmatisée par le groupe dominant en raison d'une religion et d'une origine différentes (26 articles publiés avant le 11 septembre associent le mot immigrants à des dérivés des mots "musulman » ou "Moyen-Orient »), cette catégorie de la population l'est encore davantage après le 11 septembre ( 43 articles font état de la même association sur la période concernée). Pire encore, si l'on regarde les articles qui mentionnent à la fois le terme immigrants, les termes associés au mot «terrorisme » et les termes associés aux mots « musulman » ou "Moyen-Orient », on note un seul article avant le 11 septembre 2001, mais 24 articles après. Quel que soit le discours tenu, on peut donc affirmer qu'une telle association contribue à renforcer l'idée selon laquelle le terrorisme est lié aux immigrants musulmans et/ou du Moyen-Orient.

27 Cependant, seule une analyse en détail des articles publiés nous permet de mettre en évidence le « paradoxe » de The Australian, c'est-à-dire un discours qui, contrairement à ce que l'on pourrait attendre, se fait beaucoup plus modéré après le 11 septembre en ce qui concerne ceux présentés comme le principal problème relatif à la politique australienne d'immigration, à savoir les immigrants dits «illégaux». Ainsi, si l'on regarde tout d'abord les articles publiés avant les attaques terroristes, on constate que ces derniers encouragent très nettement l'opposition du public à ce type d'immigration. À titre d'exemple, lors des manifestations et grèves de la faim qui ont eu lieu dans des centres de détention où les conditions de vie sont devenues insupportables, le journal choisit de dépeindre les immigrants comme de véritables criminels à l'encontre desquels la loi doit être plus sévère :

Trois gardiens du Centre de détention Curtin ont été agressés lorsque 200 immigrants illégaux ont mis le feu à des sanitaires et un bureau et endommagé la clôture du centre, faisant environ 250000 dollars de dégâts. [...] Cet incident fait suite à la série d'émeutes et d'évasions de plusieurs centres de détention qui a eu lieu cette année à travers le pays, notamment les émeutes qui ont impliqué environ 40 détenus jeudi dernier à Port Hedland. [...] Avec la nouvelle loi, les détenus risqueraient jusqu'à trois ans de prison pour fabrication, détention et distribution d'armes, et jusqu'à cinq ans pour tentative d'évasion. De plus, le personnel des centres de détention aurait le pouvoir de fouiller au corps les détenus (sauf fouille interne) et d'examiner ce qu'apportent les visiteurs. ${ }^{40}$

De nombreux articles mettent en avant les liens que les immigrants illégaux peuvent avoir avec des criminels de guerre ${ }^{41}$, ou le crime organisée ${ }^{42}$, et en particulier le trafic de drogue ${ }^{43}$. Le coût de leur détention et de l'examen de leur dossier est également mentionné : The Australian choisit de rapporter les mots du ministre de l'Immigration, Philip Ruddock, qui le qualifie d'« obscène $»^{44}$, et il souligne dans un autre article que 
les détenus qui font appel des décisions rendues par la justice vont probablement coûter 20 millions de dollars au contribuable durant l'année fiscale à venir ${ }^{45}$.

Contrairement à cela, le tout premier article concernant les immigrants publié après le 11 septembre choisit de dénoncer l'amalgame qui peut être fait entre réfugiés et terroristes par le biais d'une argumentation sans faille : premièrement, « ce ne sont pas les extrémistes islamistes qui tentent d'entrer en Australie, mais précisément ceux qui les fuient» (le journaliste établit d'ailleurs un parallèle avec la situation des Juifs pendant la Seconde Guerre mondiale). Ensuite, même si les attaques terroristes ont été perpétrées par des musulmans, cela ne doit pas conduire la population à conclure que tous les musulmans sont extrémistes. Enfin, l'histoire a montré la capacité de l'Australie à absorber des immigrants très différents, c'est pourquoi l'auteur espère que le pays "aura le courage de défendre une approche plus généreuse pour aider les victimes des comportements pouvant conduire à cette folie qui a frappé les ÉtatsUnis. $»^{46}$ Les demandeurs d'asile semblent ainsi avoir troqué leur statut de criminels contre celui de victimes, et le journal raconte désormais comment ces derniers bravent tous les dangers pour atteindre les côtes australiennes au péril de leur vie. L'histoire de ce couple ayant réussi à échapper à la dictature de Saddam Hussein et qui a perdu ses deux filles lorsque le bateau censé les emmener en Australie a coulé ${ }^{47}$ ou celle de ces deux femmes décédées dans des circonstances similaires ${ }^{48}$ sont davantage destinées à émouvoir le public plutôt qu'à le dresser contre ces demandeurs d'asile. Ce public est d'ailleurs âprement critiqué par Phillip Adams, qui ironise d'une part sur les Australiens ouvertement racistes et dénonce d'autre part ceux qui déguisent des sentiments similaires :

Le racisme de ces gentilles personnes qui vivent dans leurs jolies maisons et leurs jolis quartiers et disent de gentilles choses à leurs gentils amis est aussi subtil et de bon goût que leurs meubles et leurs objets d'art. Tout comme les mets qu'ils apportent à leurs soirées, il est fait des meilleurs ingrédients. Il arrive sur mon bureau enveloppé dans plusieurs couches d'une prose parfaite [...] Le fait que des centaines et des centaines de gens - dont beaucoup de femmes et d'enfants désespérés - meurent noyés n’a aucun impact sur la sérénité de ces gentilles personnes. Il y a quelque chose d'absolument monstrueux dans l'indifférence des gentils vis-à-vis de la souffrance humaine. ${ }^{49}$

Le changement de discours après le 11 septembre peut également être perçu au niveau de l'attitude du journal vis-à-vis du gouvernement en place. Même si certains journalistes continuent à soutenir la politique du Premier ministre John Howard et de son ministre de l'Immigration Philip Ruddock vis-à-vis des immigrants ${ }^{50}$, la critique se fait de plus en plus forte. La rhétorique de Ruddock, qui dépeint les boat people comme des tricheurs cherchant à court-circuiter la file d'attente formée par les autres demandeurs d'asile, est démontée avec pertinence : compte tenu du nombre de dossiers retenus par les services d'immigration (1 sur 17 en moyenne), cette "file d'attente " s'apparente davantage à un ticket de loterie, et on ne peut blâmer les réfugiés qui essaient d'entrer en Australie par d'autres moyens ${ }^{51}$. D'autres articles s'en prennent à Howard, et décrivent sa politique d'immigration comme un échec ${ }^{52}$, ou bien une honte ( " J'ai honte de notre gouvernement, de la manière ignoble dont il traite les réfugiés, et de sa détermination à polariser l'opinion publique contre eux $\left.»^{53}\right)$. Le journal défend ceux qu'il décrivait auparavant comme des «immigrants illégaux» et dénonce un gouvernement qui les "diabolise ». Ce dernier terme reviendra d'ailleurs à plusieurs reprises dans le discours post-11 septembre: seul 1 article emploie à la fois le mot immigrants et un mot dérivé de "diabolisation» avant le 11 septembre, tandis que la 
même association se retrouve dans 12 articles après le 11 septembre. Il est également intéressant de noter que The Australian essaie désormais d'éviter la référence aux immigrants "illégaux ", préférant les termes "réfugiés ", "demandeurs d'asile » ou "boat people»: si l'on regarde les articles employant ces derniers termes (conjointement avec le mot immigrants) tout en omettant soigneusement l'expression illegal immigrants, on obtient 15 articles avant le 11 septembre, mais 51 articles après. Un article insiste même sur la terminologie à employer, son auteur défendant la position selon laquelle ces immigrants ne peuvent être considérés comme illégaux que lorsqu'ils ont été rejetés par les services d'immigration ${ }^{54}$. Autrement dit, ils doivent bénéficier de la présomption d'innocence.

La comparaison des articles publiés avant et après le 11 septembre 2001 dans The Globe and Mail et The Australian nous montre que s'il y a un changement de discours tout à fait évident après le 11 septembre 2001, les caractéristiques de ce changement sont très différentes dans chacun des pays. Certes, dans les deux cas, on constate une association entre les immigrants et la menace terroriste. Au Canada, cette association a pour conséquence une critique de plus en plus forte du journal envers la politique menée par le gouvernement. Alors que ce dernier était auparavant souvent dénoncé pour son manque de compassion envers les immigrants, il ne serait désormais pas assez strict vis-à-vis du danger qu'ils représentent. The Globe and Mail, qui considérait avec sympathie les demandeurs d'asile, dépeint ensuite ces mêmes personnes comme des criminels en puissance. Ce discours contraste entièrement avec celui de The Australian: alors que le gouvernement durcit sa politique envers les immigrants illégaux, le journal critique cette attitude et semble défendre une catégorie d'immigrants trop souvent présentée, selon lui, comme des individus malveillants. Ainsi, alors qu'on aurait pu croire que les mêmes causes produiraient les mêmes effets, le changement de discours que l'on observe après le 11 septembre 2001 présente des différences radicales dans les journaux concernés, différences que les attaques terroristes ne peuvent pas, à elles seules, expliquer.

\section{Interprétation des données}

\section{Le Canada : une menace terroriste présente avant le 11 septembre}

Peut-on affirmer que le 11 septembre a été le seul facteur influençant la prise de position des journalistes en faveur d'un durcissement du contrôle des frontières ? Dans le cas du Canada, comme nous venons de le voir, le renforcement du contrôle des frontières lié au nouveau projet de loi $\mathrm{C}-11$ constituait déjà une préoccupation dominante dans les six mois précédant le 11 septembre. Et même si la critique en faveur d'un durcissement de la loi s'est faite plus acerbe dans les six mois après les attentats, le projet de loi était déjà discuté dans la Chambre des Communes et fut voté en juin $2001^{55}$, soit trois mois avant les attentats.

33 Ceci s'explique par le fait que le Canada avait déjà été la cible d'attaques terroristes auparavant, notamment à la fin des années 1990. Comme mentionné plus haut, une recherche dans les 490 articles couvrant la période entre mars 2001 et mars 2002 et associant les mots immigrants et terrorist(s) ou terrorism donne neuf réponses avant le 11 septembre, et 108 après. Une telle différence est bien évidemment due aux attentats et au lien établi entre ceux-ci et les immigrants. Toutefois, la menace était déjà 
présente avant les attaques terroristes. Un article datant du 22 octobre $2001^{56}$ recense par exemple le nombre de menaces terroristes qu'a connues le Canada sur son territoire, en se penchant plus particulièrement sur l'existence de cellules terroristes à Montréal. La journaliste remonte dix ans en arrière avec le cas d'Abdella Ouzghar, un Marocain devenu citoyen canadien, accusé de fournir des passeports à des terroristes islamiques, extradé vers la France et condamné à cinq ans de prison en 2001. Elle évoque également celui de Fateh Kamel, citoyen canadien d'origine algérienne, condamné à huit ans de prison en France pour activités terroristes. Le cas le plus connu est celui d'Ahmed Ressam, un Algérien arrêté en décembre 1999 à la frontière canadoaméricaine, avec une voiture chargée d'explosifs: il prévoyait de faire exploser l'aéroport de Los Angeles et avait également planifié des attentats à Montréal.

Un article plus ancien, datant d'avril 200157, mentionne qu'Ahmed Ressam a été condamné le mois précédent pour neuf affaires de terrorisme. Il cite également le cas de Gaetano Amodeo, sur le point d'être extradé vers l'Italie pour y répondre de deux meurtres. Un autre article, datant cette fois du 26 mai $2001^{58}$, fait référence à « ceux dont le Canada veut le moins. Ils sont vos voisins, vos connaissances, et vivent tranquillement avec un grand secret: un passé teinté d'allégations de terrorisme, de crimes de guerre ou de torture. Andrew Mitrovica fait le portrait de ces indésirables que nous essayons de mettre dehors depuis des années». Cet article dresse un inventaire des individus accusés de terrorisme ou d'activités illégales dans leur pays d'origine et qui ont pu entrer légalement au Canada, mettant ainsi le pays en danger. En somme, ces articles montrent à quel point il est facile d'entrer au Canada et d'y rester : le juge Bruguière, interviewé par le Globe and Mail, admet que « le Canada est le pays occidental où il est le plus facile d'immigrer ${ }^{59}$. Déjà avant le 11 septembre, la plupart des journalistes accusaient, directement ou non, le gouvernement canadien d'avoir une politique d'immigration trop favorable envers les individus dangereux, et exprimaient donc leur soutien en faveur d'un durcissement de cette politique.

Mais, plus généralement, ces articles permettent également de resituer le vote de la Loi sur l'immigration et la protection des réfugiés dans son contexte : la menace terroriste était présente au Canada depuis des années, et elle a joué un rôle extrêmement important dans la création et le vote de la nouvelle loi en juin 2001. Par la suite, le lien très clair entre immigrants et terrorisme (dans $40 \%$ des articles écrits après le 11 septembre) est révélateur $\mathrm{du}$ changement de ton très net $\mathrm{du}$ journal. Ceci confirme la tendance préexistante et par conséquent influe sur l'opinion en faveur du passage de la loi et de l'adoption des mesures additionnelles qui sont, de l'aveu même du gouvernement, le résultat direct des attentats terroristes ayant visé les États-Unis.

Les attentats du 11 septembre n'ont donc pas été un élément déclencheur dans le durcissement de la politique d'immigration, ni dans le vote de la Loi sur l'immigration et la protection des réfugiés qui a eu lieu trois mois avant les événements. Toutefois, ils ont joué un rôle capital en ce qui concerne le regard que portent les journalistes et plus généralement l'opinion sur cette nouvelle loi: considérée en premier lieu comme trop dure, elle est pourtant critiquée par la suite car jugée pas assez stricte et ne prenant pas suffisamment en compte l'importance de la menace terroriste. Les attentats ainsi que la critique auront également un impact sur cette loi : selon le gouvernement,

Dans la foulée des attaques terroristes du 11 septembre 2001, CIC [Citoyenneté et Immigration Canada] a adopté des mesures supplémentaires destinées à protéger la santé et la sécurité des Canadiens sans pour autant compromettre la capacité du 
Canada à attirer de nouveaux arrivants dont les apports favorisent le développement économique et social du pays. ${ }^{60}$

\section{L'Australie : une menace terroriste en pleine campagne électorale}

37 Le changement de discours de The Australian suite aux attaques du 11 septembre 2001 laisse à penser qu'effectivement, l'événement a eu des conséquences non négligeables. Ceci est particulièrement évident au vu de l'augmentation du nombre d'articles faisant le lien entre immigrants et terroristes. Pourtant, nous avons vu que le journal s'appliquait justement à révéler ce lien et se montrait plutôt critique face à l'attitude d'un gouvernement qui durcit sa position vis-à-vis des réfugiés. Cette tendance est plus difficile à interpréter à l'aune des attaques terroristes, mais on peut en revanche trouver une explication si l'on se penche sur le contexte dans lequel les événements du 11 septembre surviennent. L'Australie est en effet au milieu d'une campagne électorale qui oppose le Premier ministre libéral John Howard, au pouvoir depuis 1996, à son opposant travailliste Kim Beazley. Cette campagne va se jouer autour du thème de l'immigration, qui n'est certes pas nouveau mais qui va être relancé par deux événements majeurs. Le premier est la crise provoquée en août 2001 par le Tampa, ce navire ayant secouru des immigrants clandestins qu'il souhaite débarquer sur le sol australien. Le Tampa représente tout ce qui fait peur aux Australiens de souche : à travers lui, ils voient le spectre de ces milliers d'immigrants illégaux qui menacent la souveraineté australienne et dont la détention coûte des millions au contribuable. Le rédacteur de la rubrique politique ne s'y trompe pas lorsqu'il affirme que John Howard est tout à fait conscient du fait que "tandis que se déploie la crise du Tampa, les électeurs se forgent une opinion sur le candidat qu'ils vont choisir ${ }^{61}$. Le nombre des articles associant le terme immigrants à des termes liés aux élections (election(s) / vote(s) / voter(s) / campaign) passe d'ailleurs de 44 avant le 11 septembre à 79 après, alors que la campagne bat son plein. Le deuxième événement qui contribue à faire de l'immigration un thème central des élections est la menace terroriste liée aux attentats du 11 septembre. Cette menace pose la question du soutien que peut (ou doit) apporter l'Australie aux États-Unis, mais aussi des mesures à adopter pour éviter de telles attaques dans le pays. Parmi ces mesures, le renforcement des contrôles aux frontières paraît primordial. Ainsi, non seulement la campagne électorale ne peut ignorer ce nouveau facteur qu'est le terrorisme (on retrouve une association entre des termes liés à l'élection et des termes liés au terrorisme dans 856 articles après le 11 septembre, contre 48 seulement avant), mais ce dernier contribue également à focaliser d'autant plus l'élection sur le thème de l'immigration. Ainsi, seul un article mentionnait à la fois des termes liés à l'élection, des termes liés au terrorisme et le mot immigrants avant le 11 septembre, tandis que ce chiffre passe à 22 après le 11 septembre.

Face à cet état de fait et à une peur croissante de la population qui réclame des mesures contre l'immigration clandestine, le gouvernement Howard joue la carte de la fermeté en refusant l'entrée des immigrants du Tampa et en annonçant une politique toujours plus stricte pour limiter l'immigration «illégale». Son principal slogan de campagne, "Nous seuls décidons des personnes qui viennent dans ce pays et des circonstances dans lesquelles elles viennent » indique clairement sa position. Devant l'enjeu électoral, et compte tenu de l'opinion du public australien, les autres partis ne peuvent que suivre la donne. Avant même les attentats du 11 septembre, et dans le contexte de la crise du Tampa, le journal note ainsi : 
Que propose le gouvernement? Une position ferme : le ministre de l'Immigration Philip Ruddock s'est engagé à poursuivre et renvoyer chez eux les faux réfugiés, et le gouvernement a débloqué 124 millions de dollars supplémentaires pour renforcer la protection des frontières.

Et les travaillistes? Les travaillistes sont en faveur de la détention des immigrants illégaux, mais demandent une enquête sur la gestion des centres de détention pour s'assurer que les réfugiés ne sont pas victimes de mauvais traitements.

Quelle est la position des autres partis ? Les démocrates sont opposés à la détention obligatoire et décrivent les centres de détention comme des camps de concentration. Les Verts souhaitent que le personnel des centres de détention bénéficie d'une formation interculturelle et que les critères relatifs au statut de réfugié soient changés. One Nation est en faveur de la déportation immédiate de tout demandeur d'asile qui n'a pas de raison valable. ${ }^{62}$

On note certes l'opposition des démocrates face à la politique gouvernementale, mais en revanche les principaux partis, et en particulier les travaillistes, soutiennent cette politique, même si c'est parfois avec prudence. Cette tendance se confirme après les événements du 11 septembre, puisque le Parti travailliste offre son appui au parti de John Howard en votant pour de nouvelles lois encore plus strictes vis-à-vis des immigrants clandestins. Un article du 19 septembre 2001 indique ainsi que les travaillistes ne sont plus opposés, comme ils l'étaient depuis 1998, à une restriction du droit pour les immigrants à faire appel d'une décision d'un tribunal, et proposent même de punir les avocats qui ralentissent le travail de la justice en multipliant le nombre de ces appels par le biais d'une amende pouvant aller jusqu'à 10000 dollars ${ }^{63}$.

On comprend que l'enjeu de l'élection n'ait pas échappé aux analystes politiques, qui dès lors vont mettre à jour les manipulations auxquelles ont recours les différents partis pour gagner des voix, telle l'histoire des enfants jetés par-dessus bord ${ }^{64}$. On remarque d'ailleurs qu'après le 11 septembre, c'est-à-dire au moment où se joue l'élection prévue pour le 10 novembre, les auteurs des articles contenant le mot immigrants sont de plus en plus fréquemment de "grands noms» du journal (rédacteurs des rubriques politiques, affaires nationales ou affaires étrangères) plutôt que les personnes traitant habituellement de ce thème. Ce sont ces auteurs qui vont dénoncer la tactique de « diabolisation » des immigrants clandestins utilisée dans le but de tirer parti d'une peur de l'Autre exacerbée par les événements du 11 septembre et qui, par contraste, vont adopter un discours différent au sujet de ces mêmes immigrants et dénoncer, par exemple, la "stratégie froidement calculée $»^{65}$ de Howard en ce qui concerne l'affaire du Tampa. Ce changement de discours n'aura pourtant guère de prise sur les électeurs qui, dans ce contexte, vont choisir de voter pour le parti présentant la position la plus dure vis-à-vis des immigrants : en novembre 2001, les libéraux sont réélus avec une majorité encore plus grande qu'en 1998, et ceci, de l'avis des analystes, grâce à leur fermeté par rapport à l'enjeu principal de cette élection : la politique d'immigration.

41 Les attentats de septembre 2001 ont eu pour conséquence, au Canada et en Australie comme ailleurs, la montée d'un sentiment anti-immigration parmi la population. Cependant, alors que le discours de The Globe and Mail se fait de plus en plus le reflet de ce sentiment, celui de The Australian tente de démontrer son caractère irrationnel. Dans le premier cas, le journal canadien se focalise sur une proposition de loi destinée à renforcer la protection des frontières contre l'immigration illégale, dans le second, le journal australien analyse la réaction du gouvernement à la crise du Tampa comme emblématique d'un durcissement de la politique d'immigration en faveur duquel 
l'ensemble des principaux partis semble se prononcer dans le contexte de la campagne électorale. Les deux quotidiens ont en commun une critique du pouvoir en place: The Globe and Mail l'encourage à davantage de fermeté tandis que The Australian cherche à lutter contre l'exploitation qu'il fait de la peur des immigrants exprimée par la population.

On pourra souligner au passage l'importance du titre des articles, dont l'impact sur le lecteur est évidemment à prendre en compte. Ainsi, The Globe and Mail ne peut qu'accroître la suspicion qu'éprouvent les Canadiens vis-à-vis des immigrants illégaux lorsqu'il les décrit avant le 11 septembre comme "Canada's least wanted» ou "Canada's underground.$^{66}$ Ces mêmes lecteurs verront ensuite la menace se rapprocher au point de devenir presque banale, puisque le journal titre «Terrorism and banality in Montreal ${ }^{67}$. D'autres titres sont révélateurs d'une attitude critique visà-vis de l'Australie ("Australia snubs refugees " ${ }^{68}$ ), qui refuse de se conformer au mythe américain de la terre d'accueil (" None is too many. Don't give us your tired, your poor, your huddled masses, rich nations like Australia are telling refugees $\left.{ }^{69}\right)$. Il est vrai qu'avant le 11 septembre, cette dernière brandit elle aussi les multiples menaces liées aux immigrants illégaux («People-trade syndicates make millions $»^{70}$; «Detention facilities filling fast $\left.\aleph^{71}\right)$. Pourtant, conformément à ce que nous avons analysé plus haut, les titres des articles australiens publiés après le 11 septembre font appel à la sensibilité du lecteur (" Photo all that remains of little girls »" ; «Refugees drown as boat left burning ${ }^{73}$ ) ou critiquent ouvertement l'attitude du gouvernement et du public en général (« Muslim-bashing compounds the terror $»^{74}$; «Toxic rhetoric blurs

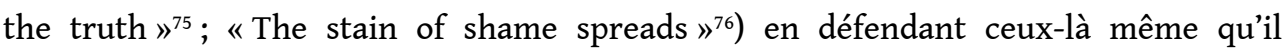
dénonçait auparavant.

Pour conclure, nous retiendrons que dans The Globe and Mail tout comme The Australian, on constate bel et bien un changement du discours après le 11 septembre 2001. En revanche, on ne peut analyser ce changement uniquement à travers cet événement puisque d'une part, ce dernier vient se superposer à un discours dominant qui présente un certain nombre de constantes sur les deux périodes analysées (avant / après le 11 septembre), et que d'autre part le contexte dans lequel cet événement s'inscrit dans chaque pays est différent, ce qui explique la nature du changement en question. On retiendra donc le danger qu'il peut y avoir à vouloir utiliser le 11 septembre comme grille de lecture universelle même si, indéniablement, son impact sur des pays comme le Canada ou l'Australie a été et reste tangible.

\section{ANNEXES}

\section{Sélection d'articles et statistiques}

The Globe and Mail

Articles publiés entre le 11 mars 2001 et le 11 septembre 2001 : 
«New immigration law called draconian, enforcement officers to be given power of 'secret police', bar association charges ", Campbell Clark, 16 mars 2001.

"What does Canada want Canada to be ? ", Editorial, 19 mars 2001.

« Refugees facing legal-aid 'crisis'. Lawyers say many will go into hiding », Kirk Makin, 21 mars 2001.

"Canada takes lead in battle against TB. Offers to set up global drug system », André Picard, 23 mars 2001.

«HIV and immigration », Editorial, 2 avril 2001.

«Stowaways lived in filthy squalor. Illegal Chinese immigrants hid on board ship heading to Long Beach, Calif. », Caroline Alphonso, 12 avril 2001.

"We're tough and we're porous ", Hugh Winsor, 18 avril 2001.

" Immigration bill will be softened », Campbell clark, 8 mai 2001.

"Caplan, family at odds over $\$ 50$ expulsion », Kirk Makin, 17 mai 2001.

«200,000 may be in Canada illegally. Economic underclass faces bleak future, but now everyone supports amnesty », Peter Cheney et Colin Freeze, 26 mai 2001.

"Canada's least wanted. They are your neighbours and your acquaintances, living quietly with a big secret : a past tainted by allegations of terrorism, war crimes or torture. Andrew Mitrovica profiles the undesirables we have been trying to kick out for years ", Andrew Mitrovica, 26 mai 2001.

"Canada's underground : illegal immigrants' stories differ in detail, but poverty and uncertainty run deep in all », Peter Cheney et Colin Freeze, 26 mai 2001.

«Denied one last hearing », Editorial, 26 mai 2001.

« Reality hard for Polish family », Kirk Makin, 29 mai 2001.

" Time to weigh amnesty », Campbell Clark, 29 mai 2001.

« Keep our door open - Immigrants and refugees to this country are getting a bad rap, says sociologist Morton Weinfield», Morton Weinfield, 22 juin 2001.

" Inkeeper called aide in terrorist bombings, shootings », John Saunders, 5 juin 2001.

«We could send back the next Mandela. Some refugees could arbitrarily be deported to face torture and prison under Canada's new legislation, say human-rights advocates ", Sharryn Aiken and Andrew Brouwer, 7 juin 2001.

« Immigration policy blamed for plight of child in Australia » Belinda Goldsmith, 15 août 2001.

« Australia snubs refugees. Fatigued asylum-seekers, who set sail from Indonesia, now refusing food and medicine », Michael Perry, 28 août 2001.

« None is too many. Don't give us your tired, your poor, your huddled masses, rich nations like Australia are telling refugees ", Marcus Gee, 30 août 2001.

\section{Articles publiés entre le 12 septembre 2001 et le 11 mars 2002 :}

"Security, Intelligence, potential toll to dominate cabinet discussion today ", Campbell Clark, 18 septembre 2001.

«Immigrant or terrorist? ?, Editorial, 19 septembre 2001.

« Canada and U.S. tighten borders - Ashcroft orders more security - Caplan toughtens refugee screening - Air Canada staff brace for layoffs - Taliban blames U.S. for attacks ", John Ibbitson et Campbell Clark, 26 septembre 2001.

" Most want PM to cede sovereignty over border ", André Picard, ${ }^{\text {er }}$ octobre 2001.

«What are Ottawa's plans to tighten immigration rules?", Estanislao Ozeiwiz,

3 octobre 2001.

«A more secure border must suit Canada first », Janet McFarland, 6 octobre 2001. 
«Immigration security : from bad to worse. Our new immigration bill poses an even greater risk of abuse by asylum seekers than the old one, says James Bissett. After Sept. 11, that's a scandal », James Bissett, 16 octobre 2001.

« Tighten immigration laws, Ottawa told», Shawn McCathy, 17 octobre 2001.

« Terrorism and banality in Montreal », Lysiane Gagnon, 22 octobre 2001.

«The politics of a secure border », Editorial, 31 octobre 2001.

« How the refugee system got undermined », Margaret Wente, 3 novembre 2001.

«Martin's security blanket 'Our purpose is clear : to keep Canadians safe, to keep terrorists out and to keep our borders open'», Shawn McCarthy, 11 décembre 2001. «Our planes will be safer and our border more secure. But we still need manpower, national standards and an anthrax plan, expert says », Jan Wong, 11 décembre 2001. « Tougher immigration screening attacked », Daniel Leblanc, 22 décembre 2001.

«Immigrant screening seen as lax, poll says. But most Canadians approve security steps taken by Ottawa to protect airports, planes and border crossings, new survey indicates ", Steven Chase, 26 décembre 2001.

" Is new legislation needed to address security risks ? ", Campbell Clark, 22 janvier 2002 .

« Detainees protest Australian policy », Roma Luciw, 22 janvier 2002.

« Al-Qaeda suspect watched, witness account suggest », Tu Thanh Ta, 28 janvier 2002.

"Would-be immigrants caught in switch of rules could get fee refunds », Campbell

Clark, 30 janvier 2002.

"Pakistanis, Afghans finding visas elusive for entry to Canada », Mark MacLinnon, $1^{\text {er }}$ février 2002.

« Police probe charges of people smuggling. Allegations of crime ring using false papers for illegals being checked, Codere says », Jeff Sallot, 1er mars 2002.

Tableau récapitulatif des statistiques (les pourcentages sont donnés en fonction du nombre total d'articles contenant le mot immigrants) :

\begin{tabular}{|c|c|c|}
\hline Expression recherchée & $\begin{array}{c}\text { Du } 11 \text { mars } 2001 \\
\text { au } 11 \text { septembre } \\
2001\end{array}$ & $\begin{array}{c}\text { Du } 12 \text { septembre } \\
2001 \\
\text { au } 11 \text { mars } 2002\end{array}$ \\
\hline Immigrants & 222 & 268 \\
\hline Illegal immigrants & $33(15 \%)$ & $52(19 \%)$ \\
\hline Immigrants AND [terrorist OR terrorists OR terrorism] & $9(4 \%)$ & $108(40 \%)$ \\
\hline $\begin{array}{l}\text { Illegal immigrants AND [terrorist OR terrorists } O R \\
\text { terrorism] }\end{array}$ & 0 & $27(10 \%)$ \\
\hline Immigrants AND [asylum seeker OR asylum seekers] & $6(<3 \%)$ & $6(<3 \%)$ \\
\hline Immigrants AND [refugee OR refugees] & $38(17 \%)$ & $81(30 \%)$ \\
\hline Illegal immigrants AND crime & $4(<2 \%)$ & $6(<3 \%)$ \\
\hline Illegal immigrants AND [criminal or criminals] & $5(<3 \%)$ & $8(3 \%)$ \\
\hline
\end{tabular}




\begin{tabular}{|l|l|l|}
\hline Refugees AND crime & 18 & 16 \\
\hline Refugees AND [criminal or criminals] & 15 & 21 \\
\hline
\end{tabular}

The Australian

\section{Articles publiés entre le 11 mars 2001 et le 11 septembre 2001}

« Ruddock won't give in to rioters », Megan Saunders, 6 avril 2001. « 6,000 new migrant vacancies », Claire Harvey, 28 avril 2001.

«Cost of illegal immigrants obscene, says Ruddock », Megan Saunders, 1er mai 2001.

« Refugee status under fire - Ruddock to rein in 'too generous' judges », Megan Saunders, 7 mai 2001.

" Raid grabs refugees' weapons ", Belinda Hickman, Christina Cridland et Megan Saunders, 28 mai 2001.

« Let illegals out after 14 weeks - MPs », Megan Saunders, 19 juin 2001.

« People who move people », Don Greenlees, 23 juin 2001.

« People-trade syndicates make millions », Don Greenlees, 23 juin 2001.

«The brain gain counters drain », Megan Saunders, 20 juillet 2001.

«E-tags plan to replace detention », Megan Saunders, 24 juillet 2001.

« Hopes blown out of water », Leisa Scott et Claire Harvey, 18 août 2001.

« Detention facilities filling fast », Megan Saunders, 22 août 2001.

"Coastwatch 'blind spots' anger ALP », Megan Saunders, 23 août 2001.

"Stern gatekeeper sees kindness in apparent cruelty », Megan Saunders, 28 août 2001.

« First step - a deal with Indonesia », Paul Kelly, 29 août 2001.

«Business booming for gang of six », Megan Saunders, 31 août 2001.

« Howard courts talkback nation », Dennis Shanahan, 31 août 2001.

«Let hard heads, not halfwits, deal with illegals ", Frank Devine, 3 septembre 2001.

«Bin Laden code red in Jakarta », Don Greenlees, 4 septembre 2001.

« Fresh blood keeps the country young », Mike Steketee, 6 septembre 2001.

«We of never land», Paul Kelly, 8 septembre 2001.

\section{Articles publiés entre le 12 septembre 2001 et le 11 mars 2002}

« Muslim-bashing compounds the terror », Mike Steketee, 14 septembre 2001.

« Labor reverses illegal policy », Megan Saunders et Ian Henderson, 19 septembre 2001.

« Toxic rhetoric blurs sorry truth», Mike Steketee, 25 septembre 2001.

« Reception committee for people-smuggler », Don Greenlees, 8 octobre 2001.

« Deal with PNG to take boatpeople », Megan Saunders et Natalie O’Brien, 12 octobre 2001.

« Beazley targets Indonesia ties - Election 2001 », Robert Garran et John Kerin, 17 octobre 2001.

« Wets freeze up in Howard's way », Mike Steketee, 17 octobre 2001.

"Photo all that remains of little girls », Don Greenlees et Vanessa Walker, 25 octobre 2001.

« Muslims say PM to blame for tragedy », Penny Brown et Claire Harvey, 26 octobre 2001.

« Make the world go away », Greg Sheridan, 27 octobre 2001.

«Virtuous outrage takes the wrong line », Frank Devine, 1er novembre 2001.

"The stain of shame spreads », Greg Sheridan, 8 novembre 2001. 
«Refugees drown as boat left burning », Robert Garran et Megan Saunders, 10 novembre 2001.

« People by definition », Frank Devine, $1^{\text {er }}$ décembre 2001.

«A nice line in bigotry », Phillip Adams, $1^{\text {er }}$ décembre 2001.

"And you, sir, are not Bush », Greg Sheridan, 27 décembre 2001.

« Detention remains in ALP policy », Dennis Shanahan et Nicole Strahan, 6 février 2002.

«Woomera - we've been here before », Dennis Shanahan, 8 février 2002.

«Butt out of it, bishops », Angela Shanahan, 12 février 2002.

« Positively the way to go for the ALP », Dennis Shanahan, 15 février 2002.

«Growth must come from within », Angela Shanahan, 26 février 2002.

Tableau récapitulatif des statistiques (les pourcentages sont donnés en fonction du nombre total d'articles contenant le mot immigrants) :

\begin{tabular}{|c|c|c|}
\hline Recherche & $\begin{array}{l}11 \text { mars } 2001 \\
\text { au } 11 \text { septembre } \\
2001\end{array}$ & $\begin{array}{c}12 \text { septembre } \\
2001 \\
\text { au } 11 \text { mars } \\
2002\end{array}$ \\
\hline immigrants & 172 & 217 \\
\hline illegal immigrants & $105(61 \%)$ & $101(46 \%)$ \\
\hline $\begin{array}{l}\text { immigrants AND [refugee OR refugees OR boatpeople OR boat } \\
\text { people OR asylum seeker OR asylum seekers] }\end{array}$ & $79(46 \%)$ & $117(54 \%)$ \\
\hline $\begin{array}{l}\text { illegal immigrants AND [refugee OR refugees OR boatpeople OR } \\
\text { boat people OR asylum seeker OR asylum seekers] }\end{array}$ & $64(37 \%)$ & $66(30 \%)$ \\
\hline $\begin{array}{l}\text { immigrants AND [smuggler OR smugglers OR smuggling OR } \\
\text { people-smuggler OR people-smugglers OR people-smuggling] }\end{array}$ & $36(21 \%)$ & $40(18 \%)$ \\
\hline immigrants AND [detention OR detained] & $54(31 \%)$ & $51(24 \%)$ \\
\hline immigrants AND [terrorism OR terrorist OR terrorists] & $2(1 \%)$ & $53(24 \%)$ \\
\hline $\begin{array}{l}\text { immigrants AND [Muslim OR Muslims OR Middle-East OR Mid- } \\
\text { East OR Middle-Eastern] }\end{array}$ & $26(15 \%)$ & $43(20 \%)$ \\
\hline $\begin{array}{l}\text { immigrants AND [Muslim OR Muslims OR Middle-East OR Mid- } \\
\text { East OR Middle-Eastern] AND [terrorism OR terrorist OR terrorists] }\end{array}$ & $1(<1 \%)$ & $12(6 \%)$ \\
\hline $\begin{array}{l}\text { immigrants AND [demoni ?ation OR demoni ?ed OR demoni ?e OR } \\
\text { demoni ?es OR demoni ?ing] }\end{array}$ & $1(<1 \%)$ & $12(6 \%)$ \\
\hline $\begin{array}{l}\text { immigrants AND [refugee OR refugees OR boatpeople OR boat } \\
\text { people OR asylum seeker OR asylum seekers] NOT illegal } \\
\text { immigrants }\end{array}$ & $15(9 \%)$ & $51(24 \%)$ \\
\hline $\begin{array}{l}\text { immigrants AND [election } O R \text { elections } O R \text { vote } O R \text { votes } O R \text { voter } \\
\text { OR voters } O R \text { campaign] }\end{array}$ & $44(26 \%)$ & $79(36 \%)$ \\
\hline
\end{tabular}




\begin{tabular}{|l|l|l|}
\hline $\begin{array}{l}\text { immigrants AND [terrorism OR terrorist OR terrorists] AND } \\
\text { [election OR elections OR vote OR votes OR voter OR voters OR } \\
\text { campaign] }\end{array}$ & $1(<1 \%)$ & $22(10 \%)$ \\
\hline
\end{tabular}

\section{NOTES}

1. Ces attentats, bien qu'ayant eu lieu en Indonésie, ont fait une majorité de victimes australiennes.

2. Utilisation de la base de données Factiva.

3. «Immigrant or terrorist? ", Éditorial Globe and Mail, 19 septembre 2001.

4. «Immigration bill will be softened », Campbell Clark, Globe and Mail, 8 mai 2001.

5. "Immigration policy blamed for plight of child in Australia », Belinda Goldsmith, Globe and Mail, 15 août 2001.

6. « None is too many. Don't give us your tired, your poor, your huddled masses, rich nations like Australia are telling refugees », Marcus Gee, Globe and Mail, 30 août 2001.

7. Ibidem.

8. Ibid.

9. Si on exclut The Australian Financial Review, qui s'adresse davantage au monde des affaires.

10. Phillip Adams a notamment publié un recueil d'articles critiques sur la société australienne après l'élection au Parlement australien de Pauline Hanson, à la tête du parti d'extrême droite One Nation. Phillip Adams (ed.), The Retreat from Tolerance. A snapshot of Australian society, Sydney: ABC Books, 2001.

11. Auteur d'un livre sur l'alliance entre les États-Unis et l'Australie. Robert Garran, True Believer : John Howard, George Bush and the American alliance, Sydney : Allen and Unwin, 2004.

12. «6,000 new migrant vacancies », Claire Harvey, The Australian, 28 avril 2001.

13. Ibid.

14. Recherche effectuée à partir des mots «smuggler(s)», "smuggling », «people-smuggler(s)» et «people-smuggling».

15. «People who move people », Don Greenlees, The Australian, 23 juin 2001.

16. «First step: a deal with Indonesia », Paul Kelly, The Australian, 29 août 2001.

17. «Reception committee for people-smuggler », Don Greenlees, The Australian, 8 octobre 2001.

18. «People who move people », Don Greenlees, The Australian, 23 juin 2001.

19. «Beazley targets Indonesia ties-Election 2001 », Robert Garran et John Kerin, The Australian, 17 octobre 2001.

20. "Let hard heads, not halfwits, deal with illegals ", Frank Devine, The Australian, 3 septembre 2001.

21. «E-tags plan to replace detention », Megan Saunders, The Australian, 24 juillet 2001.

22. "Detention facilities filling fast ", Megan Saunders, The Australian, 22 août 2001.

23. Sans doute ici en référence à l'article publié par Phillip Adams, "A nice line in bigotry ", The Australian, $1^{\text {er }}$ décembre 2001.

24. "Growth must come from within ", Angela Shanahan, The Australian, 16 février 2002.

25. " Hopes blown out of water », Leisa Scott et Claire Harvey, The Australian, 18 août 2001.

26. "New immigration law called draconian, enforcement officers to be given power of 'secret police', bar association charges », Campbell Clark, Globe and Mail, 16 mars 2001.

27. «Immigration bill will be softened », Campbell Clark, Globe and Mail, 8 mai 2001. 
28. «Our planes will be safer and our border more secure. But we still need manpower, national standards and an anthrax plan, expert says ", Jan Wong, Globe and Mail, 11 décembre 2001.

29. «Denied one last hearing ", Editorial, Globe and Mail, 26 mai 2001.

30. "Inkeeper called aide in terrorist bombings, shootings », John Saunders, 5 juin 2001.

31. «Canada's underground: illegal immigrants' stories differ in detail, but poverty and uncertainty run deep in all », Peter Cheney et Colin Freeze, Globe and Mail, 26 mai 2001.

32. Ibid.

33. "Immigration security: from bad to worse. Our new immigration bill poses an even greater risk of abuse by asylum seekers than the old one, says James Bissett. After Sept. 11, that's a scandal », James Bissett, Globe and Mail, 16 octobre 2001.

34. « Keep our door open-Immigrants and refugees to this country are getting a bad rap, says sociologist Morton Weinfield », Morton Weinfield, Globe and Mail, 22 juin 2001.

35. «Immigration security: from bad to worse. Our new immigration bill poses an even greater risk of abuse by asylum seekers than the old one, says James Bissett. After Sept. 11, that's a scandal », James Bissett, Globe and Mail, 16 octobre 2001.

36. "How the refugee system got undermined ", Margaret Wente, Globe and Mail, 3 novembre 2001.

37. "Most want PM to cede sovereignty over border ", André Picard, Globe and Mail, $1^{\text {er }}$ octobre 2001.

38. «Canada and U.S. tighten borders - Ashcroft orders more security - Caplan toughtens refugee screening - Air Canada staff brace for layoffs - Taliban blames U.S. for attacks ", John Ibbitson and Campbell Clark, Globe and Mail, 26 septembre 2001.

39. "A more secure border must suit Canada first ", Janet McFarland, Globe and Mail, 6 octobre 2001.

40. «Ruddock won't give in to rioters », Megan Saunders, The Australian, 6 avril 2001.

41. "Cost of illegal immigrants obscene, says Ruddock », Megan Saunders, The Australian, $1^{\mathrm{er}}$ mai 2001.

42. "Refugee status under fire-Ruddock to rein in 'too generous' judges », Megan Saunders, The Australian, 7 mai 2001.

43. «Coastwatch 'blind spots' anger ALP », Megan Saunders, The Australian, 23 août 2001.

44. "Cost of illegal immigrants obscene, says Ruddock », Megan Saunders, The Australian, $1^{\mathrm{er}}$ mai 2001.

45. «Raid grabs refugees' weapons ", Belinda Hickman, Christina Cridland et Megan Saunders, The Australian, 28 mai 2001.

46. "Muslim-bashing compounds the terror ", Mike Steketee, The Australian, 14 septembre 2001.

47. "Photo all that remains of little girls", Don Greenlees et Vanessa Walker, The Australian, 25 octobre 2001.

48. "Refugees drown as boat left burning ", Robert Garran et Megan Saunders, The Australian, 10 novembre 2001.

49. "A nice line in bigotry ", Phillip Adams, The Australian, $1^{\mathrm{er}}$ décembre 2001.

50. «Growth must come from within », Angela Shanahan, The Australian, 26 février 2002.

51. "Toxic rhetoric blurs sorry truth », Mike Steketee, The Australian, 14 septembre 2001.

52. "The stain of shame spreads", Greg Sheridan, The Australian, 8 novembre 2001.

53. «And you, sir, are not Bush », Greg Sheridan, The Australian, 27 décembre 2001.

54. "People by definition », Frank Devine, The Australian, $1^{\text {er }}$ décembre 2001.

55. «Immigrant or terrorist? », Editorial, Globe and Mail, 19 septembre 2001.

56. « Terrorism and banality in Montreal », Lysiane Gagnon, Globe and Mail, 22 octobre 2001.

57. «We're tough and we're porous », Hugh Winsor, Globe and Mail, 18 avril 2001.

58. «Canada's least wanted. They are your neighbours and your acquaintances, living quietly with a big secret: a past tainted by allegations of terrorism, war crimes or torture. Andrew 
Mitrovica profiles the undesirables we have been trying to kick out for years ", Andrew Mitrovica, Globe and Mail, 26 mai 2001.

59. «Terrorism and banality in Montreal », Lysiane Gagnon, Globe and Mail, 22 octobre 2001.

60. Secrétariat du Conseil du Trésor du Canada (26 mai 2008)

61. "Howard courts talkback nation ", Dennis Shanahan, The Australian, 31 août 2001.

62. "Hopes blown out of water", Leisa Scott et Claire Harvey, The Australian, 18 août 2001.

63. "Labor reverses illegal policy», Megan Saunders et Ian Henderson, The Australian, 19 septembre 2001.

64. Juste avant l'élection, le parti libéral avait utilisé des photos montrant des immigrants clandestins jetant leurs enfants à la mer pour dire à quel point il était nécessaire de se montrer ferme par rapport à des individus capables de mettre la vie de leurs propres enfants en danger afin d'obtenir une aide. On apprendra par la suite que l'histoire et les documents avaient été fabriqués de toutes pièces.

65. «Wets freeze up in Howard's way », Mike Steketee, The Australian, 17 octobre 2001.

66. Que l'on pourrait traduire par "les plus indésirables» ou "les clandestins " du Canada. "Canada's least wanted ", Andrew Mitrovica, Globe and Mail, 26 mai 2001 ; "Canada's underground », Peter Cheney et Colin Freeze, Globe and Mail, 26 mai 2001.

67. « Terrorism and banality in Montreal », Lysiane Gagnon, Globe and Mail, 22 octobre 2001.

68. "Australia snubs refugees », Michael Perry, Globe and Mail, 28 août 2001.

69. On relèvera la référence au célèbre poème d'Emma Lazarus gravé sur le socle de la Statue de la Liberté, véritable emblème de ce mythe. "None is too many. Don't give us your tired, your poor, your huddled masses, rich nations like Australia are telling refugees", Marcus Gee, Globe and Mail, 30 août 2001.

70. «People-trade syndicates make millions », Don Greenlees, The Australian, 23 juin 2001.

71. «Detention facilities filling fast ", Megan Saunders, The Australian, 22 août 2001.

72. "Photo all that remains of little girls", Don Greenlees et Vanessa Walker, The Australian, 25 octobre 2001.

73. "Refugees drown as boat left burning ", Robert Garran et Megan Saunders, The Australian, 10 novembre 2001.

74. «Muslim-bashing compounds the terror ", Mike Steketee, The Australian, 14 septembre 2001.

75. " Toxic rhetoric blurs the truth », Mike Steketee, The Australian, 25 septembre 2001.

76. "The stain of shame spreads ", Greg Sheridan, The Australian, 27 décembre 2001.

\section{RÉSUMÉS}

Les attentats du 11 septembre 2001 ont profondément bouleversé le champ des représentations, non seulement aux États-Unis mais également au sein de pays comme le Canada ou l'Australie qui ont offert leur soutien au gouvernement américain dans sa " guerre contre la terreur ». Ainsi, la figure de l'immigrant, par exemple, est très vite apparue comme une menace potentielle pour la sécurité des citoyens. Dans le but de creuser ce constat, cet article se penche sur les transformations du discours journalistique canadien et australien au sujet des immigrants suite aux attentats. À travers l'analyse d'articles publiés dans The Globe and Mail et The Australian au cours des 6 derniers mois précédant et des 6 derniers mois suivant le 11 septembre 2001, il fait apparaître un certain nombre de constantes caractéristiques du « discours dominant » relatif aux 
immigrants. Cependant, il note également des différences très nettes au niveau du discours produit avant et après les attentats. Pour finir, il montre de quelle manière le contexte dans lequel s'inscrit ce discours dans chaque pays est, au-delà de l'impact des attentats, un élément supplémentaire qui permet d'apprécier ce changement, et donc d'éviter toute tentative de lecture unique à travers le prisme du 11 septembre.

The September 11, 2001 attacks have considerably altered existing representations, not only in the United States but also in countries such as Canada or Australia, which offered their support to the American government in its "war against terror". One consequence has been that the figure of the immigrant soon came to represent a potential threat to the security of citizens in these countries. In order to inquire into this phenomenon, this article proposes to look at transformations relating to the journalistic discourse on immigrants following the attacks. Through the analysis of articles published in The Globe and Mail and The Australian in the 6 months preceding and following these attacks, the paper underlines a certain number of features that define the "dominant discourse" related to immigrants. However, it also points at very clear differences between the kind of discourse produced before the attacks and after. To conclude, it shows how the context in which such discourse is inscribed in each country is another element which, beyond the attacks themselves, enables us to explain this change, and thus avoid looking at it through the sole prism of September 11.

\section{INDEX}

Mots-clés : attentats, 11 septembre 2001, terrorisme, discours, immigrants, immigration, immigration illégale, réfugiés, Canada, Australie, The Globe and Mail, The Australian Keywords : attacks, September 11, terrorism, discourse, immigrants, immigration, illegal immigration, refugees, Canada, Australia, The Globe and Mail, The Australian

\section{AUTEURS}

\section{SANDRINE TOLAZZI}

Université Stendhal-Grenoble 3

CEMRA

\section{CAROLE MASERATI}

Université Stendhal-Grenoble 3

CEMRA 